\title{
Acoustic Analysis of English Long Vowels Produced by Pashto Speakers
}

\author{
Adnan Ahad $^{1 *}$, Ghani Rahman ${ }^{1} \&$ Abdul Hamid ${ }^{2}$ \\ 1. Department of English, Hazara University, Mansehra, Pakistan. \\ 2. Department of English, University of Swat, Mingora, Swat, Pakistan.
}

Received: September 5, 2020

Published: December 25, 2020

\begin{abstract}
The present study investigates the acoustic properties of English long vowels produced by Pashto speakers by highlighting the problematic areas for Pashto speakers learning English. The data was collected from ten Pashtun learners of English through tape recorder. The data was analyzed using PRAAT software. The spectrograms produced by the software helped us in specifying the acoustic values (formant frequencies of F1 and F2) of target vowels compared with the production of the same vowels by native speakers of English. The analysis of the collected data revealed the problematic areas of English long vowels for Pashtun learners of English. The study proved that English long vowels like /r:/ and /a:/ were more problematic for Pashtun learners. A significant difference was seen in both the height and backness of the target English vowels produced by the subjects. The English vowels like /3:/ and /s:/ produced by subjects were like those produced by the native speakers. Similarly, target vowel like /u:/ was pronounced by subjects like native speakers of English. The study recommends that Pashto speakers should be given proper training for acquiring correct pronunciation of English.
\end{abstract}

Keywords: Problematic Vowels, L2 Learners, PRAAT, Formants, Quality of Vowels, Length of Vowels, Pronunciation, Front Long Vowel, Back Long Vowel.

\section{Introduction}

Every language has its own phonetics and phonology. Similarly, every language specifies the number of sounds to make higher units and so, is recognized from through a particular accent. Phonological proficiency in second language (English) involves the mastery of patterns of vowels (Schwartz \& Kaźmierski, 2019). English and Pashto are two different languages and therefore, have different phonological systems. English being a global language is learnt around the world as a second language and so by the Pashto speakers too. Pashto is one of the languages along other fifty-nine languages (Rahman, 2002) or seventy-two languages (Gordon, 2005) spoken in Pakistan. Every language, similarly, has different varieties in terms of grammar, pronunciation, vocabulary and usage called dialects of that language (Sethi \& Dhamija, 1999).

Different sources have different opinions about the dialects division of Pashto language. All these differences occur mainly in pronunciation. Accent variation is mostly based on amplitude, pitch, intonation and phonemes duration. By the term accent we mean the way of 
pronunciation by a speaker to which he/she belongs to. Linguistically, the accent difference does not only reside in the phonetic characterization but also in prosody (Mixdorff et al., 2012). The study of Benzeghiba et al. (2006) showed that accent is one of the striking factors that affects the automatic speech recognition system. In recent years, there has been an increasing interest of the researchers on the sound characterization of the languages from various points of view.

A considerable literature has been published on some common features across languages, while much of the researchers have concentrated on the phonetic details of a specific language. This paper aims to analyze English long vowels for Pashto speakers with English background. Therefore, in this research only long vowels were acoustically analyzed by measuring the formant frequencies: F1 and F2 of the Pashto speakers using English. These English sounds produced by Pashto speakers were also compared with the formant frequencies of Standard Southern British English. Formant frequencies account for the better understanding of accents of spoken languages. Those students who try to speak English, their language is full of phonological errors. Apart from other linguistic and phonological errors, vowels cause problem. Phonological differences for the different dialects of Pashto are primarily important than differences in other systems of the language (Mackenzie, 1959).

Similarly, when Pashto speakers learn English, they especially face problems in the productions of speech sounds. Some of these problems have been investigated in previous studies like the English problematic consonant sounds (Rehman \& Bukhari, 2012) and English short vowels (Baidar et al., 2020). The latter study focused on the quality of English short vowels only, leaving the problems in the length of the vowels for future study. The present study fills the identified gap in this regard. No one denies the fact that mispronunciation sometimes leads to misunderstanding. Consequently, a complete failure or miscommunication occurs. A change in one sound of a word may change the whole meaning of the word. The students face problems in correct pronunciation of English words due to discrepancies in the sounds system of the target language and first language.

The misinterpretation of spelling symbols also causes pronunciation problem (Schaetzel, 2009). Some students often get confused in writing of spelling. The main reason of this confusion is that students expect one to one correspondence between sound and its letter, as a result miscommunication occurs. In the same fashion, problems are faced by the Pashto speakers learning English in some areas of pronunciation. They make mistakes in pronouncing words containing long vowels which lead to miscommunication. Therefore, this research has focused on the problematic long vowels and their acoustic values for the Pashto speakers learning of the correct English language. The students confuse both the quality and quantity of vowels and as a result, they produce different sounds which lead to miscommunication and wrong comprehension.

No other studies have focused on these problems on English vowels for the Pashto speakers. Therefore, the objectives of this study are to investigate the English problematic long vowels for Pashto speakers and the acoustic nature of these long vowels. The study has delimited itself to the investigation of long vowels only ignoring other important areas of pronunciation. The study thus contributes to the body of cross-linguistic research in terms of speech sounds and the implications of the same for the English language teaching and learning. The second language teachers of English and the English language learners can benefit from the findings of this study. This study aims to find the English problematic long vowels for the Pashto 
speakers and that how are these English long vowels acoustically different from the standard English long vowels?

\section{Literature Review}

There are five long vowels in standard English which are shown in the figure-1 (annexed) along with their tongue positions. The vowels are differentiated with respect to tongue position which can vary across languages (Jia et al., 2017). There is a growing body of previous research on acoustic phonetics for the study of phonemes in many languages. The vowel sounds having persistent patterns in speech have been studied more than other speech sounds (Moore, 2003; Kent \& Charles, 2002). Some of these studies include the study of English vowels like the study of Peterson \& Barney (1952), and Chen et al. (2001). Other such studies are conducted about Indian accented English by Maxwell \& Jannet (2009) and Singapore accented English by Deterding (2003) and Japanese accented English vowels by Kewley-Porr et al. (1996). Other languages that have been studied acoustically are languages like French by Ouni \& Yves (2003), Shanghai Chinese by Chen (2008), Turkish language by Gordon \& Ayla (2006), Spanish by Borzone-de-Manrique (1979) and Borzone-de-Manrique \& Massone (1981) and Japanese by Homma (1973), and Modern Hebrew by Aronson et al. (1996).

Speech sounds in English Language have been compared with other speech sounds in other languages like Pashto (Rahman, 2009) and Spanish (Bradlow, 1995). There are other studies about pitch discrepancy, for example, Yuasa (2001) focusing on familiarity and non-familiarity of speakers, or mapping issue, i.e., the degree to which second language learners treat L2 vowel sounds equivalent to L1 vowel sounds (Bohn, 2017) and so, assuming them like L1 sounds and assimilating them (Cebrian, 2019) and the level the learners too makes difference in terms of learning the vowel system of second language (Balas, 2018). But phonological problems are not only studied in national context (different varieties of the same language) but also in international context (different varieties of different languages), giving rise to different varieties and different pronunciation (Roach, 2000) and as a result, causing sometimes unsuccessful communication. For competence in a language, many skills like the skills in vocabulary and grammar are no doubt important but pronunciation skill has utmost status (Jenkins, 2000). The studies included in the review have listed the following errors in pronunciation of speech sounds.

a) When the learners try to pronounce unfamiliar sounds nearer to the sounds, which the speaker is familiar with.

b) When learners are familiar with the sounds but they do not perceive these sounds as different sounds that make difference to meaning.

c) When learners have learned the speech sounds but have not learned the exact pattern of intonation and stress pattern.

A change in one sound in the target language may change the meaning of the entire word (Rehman \& Bukhari, 2012). The reasons for this weakness in pronunciation are lack of exposure to input in the target language, lack of motivation and attitude, influence of L1, lack of inspiration and stimulation from the teachers, problems in spelling systems, lack of proper attention to rhythm, intonation and stress and lack of required tools for teaching pronunciation (Jenkins, 2000). Learners' exposure and ability to input one of the most important and major factors in learning correct English pronunciation (Haymes, 2000) because of the phonological difference between two languages is one of the greatest reasons to impede correct 
pronunciation learning (Lin, 2014; Schwartz et al., 2016). The factors which give rise to the pronunciation errors are: a) Interference of L1; b) Lack of practice of L2; c) Being only passive learner and not active ones; d) Hesitation in speaking and using L2; e) No proper role of teacher and students in learning context; and f) No proper guidance for learners.

The commonly considered errors are phonetic error, phonemic error, distributional error, and allophonic error because of the L1 interference while learning L2 (Moulton, 1962). Moulton's taxonomy (1962) is based on understanding how these errors are made, and why they were made. Because of the influence of mother tongue, problems in L1 phonology could be easily predicted to help the learners with different linguistic backgrounds. These problems could be solved with the help of phonetic transcription of the target language (English language particularly). A practical reference guide to teachers is given as it is anticipated that the comparison between English and learners' own language would make easy for teacher to understand the problems their students have (Swan \& Smith, 2001). Vowels' acquisition is also researched from different perspectives like generative linguistics (Slabakova, 2016) and across varieties of English as a second language (Bohn, 2017; Schwartz \& Kaźmierski, 2019). The variation in speech, inherently multidimensional and varying across time has been studied for vowel variation (Roettger et al., 2019). The vowels are identified through the formant dynamics dynamics (Elvin et al., 2016).

\section{Research Methodology}

This study is concerned with English long vowels in order to put light on the problematic vowels for Pashto speakers learning English. The design for the present study is experimental which involves quantitative approach to its data analysis to base the study on more reliable and scientific bases. The pronunciation of target vowels by Pashto speakers was investigated and the most occurring problematic vowels were highlighted. The population of the present study was Pashto speakers of Yusafzai dialect with English background. To select a sample from the target population, ten native Pashto speakers (Yusafzai dialect) from the target population were selected. The sample represented the areas of the province Khyber Pakhtunkhwa, Pakistan where Yusafzai dialect is spoken. Participants include both male and female who were all graduates and they had studied English as a compulsory subject in their schools, colleges and universities. Their ages ranged from 20 to 30 years at the time of recordings. No participants had any speech disorder.

The five long vowels in the Standard English (RP) were considered problematic for the Pashto speakers of Yusafzai dialect while learning English. The subjects were given words containing target vowels at three possible different positions i.e., at word initial, medial and final positions. They were instructed to pronounce the words containing target vowels with their normal speech rate. The purpose of the instruction was to reduce the chances of errors in pronunciation. Pashto speakers (Yusafzai dialect) pronounced the selected words containing target vowels at three possible different positions i.e., at word initial, medial and final positions. The pronunciation of selected words by Pashto speakers was recorded. In this way, data was collected from participants through recording for which the researchers needed reliable data collecting tools.

A printed sheet containing words with target vowels at three possible different positions i.e., at word initial, medial and final positions was given to each participant. They pronounced the words containing target vowels. Their pronunciations were recorded. Recordings from the participants of the words containing target vowels were done with the help of laptop having 
embedded recorder and a built-in microphone. A quiet room was used for the recordings in order to avoid other noises. High quality speakers were also used in the present study in order to hear the pronunciation of participants clearly.

The data for the present study was recorded by the permission of the participants for its analysis. Recording of the pronunciations of the speakers was done in a quiet room in order to avoid other sounds of the surroundings. Additionally, only participant and the experimenter were in the room. In order to avoid confusion and ambiguity in the analysis, all the participants were asked to utter two different words having same target vowel at same position. After recording, the data was recognized and for further analysis it was processed on computer software namely PRAAT. The software PRAAT gave us spectrogram for the observation of the data. So, the pronunciation of the target vowels was completely assumed after the observation of the data through spectrogram. The spectrograms can distingue the frontness and backness of sounds (Chládková et al., 2016).

The data was heard with the help of quality speakers in order to hear the words clearly. By listening to the recorded data, the problematic vowels were recognized. The problematic vowels were heard time and again. So, the pronunciation of the most of the participants was recognized. In addition to it, the data was further processed on PRAAT software. The articulation of the problematic vowels was specified through spectrograms. The produced target vowels by Pashto speakers were compared with the Standard Southern British English vowels. The data of native vowels was taken from Deterding (1997).

\section{Results and Analysis}

According to Prica and Ilić (2010) vowels are characterized by using formants namely F1 and F2. F1 is thought to be inversely related to vowels' height. The higher the tongue position higher will be the vowel and lower will be the formant frequency (F1). F2 is thought to be somewhat related to degree of backness. The backward the tongue position, vowel will be round and higher will be the formant frequency (F2). The horizontal red lines in the figure show the formant frequencies. The horizontal red line from the bottom side of the figure shows formant F1 while the horizontal red line above formant F1 is formant F2.

\subsection{The Pronunciation of the English Front Close Long Vowel / i: /}

The table-1 below shows the pronunciation of English front close target long vowel / i: /. This long vowel was pronounced at three different possible positions of English words, i.e., at initial, medial and final positions. In the table, the leftmost column shows the different pronunciation (different realizations of a single long vowel) of the target long vowel /i:/. The rows in the table show the number of these realizations for this long vowel at different positions.

Table 1: Pronunciation of the front close target long vowel / r: /

\begin{tabular}{lccc}
\hline Pronunciation of target vowel /I:/ & The pronunciation by Pashto speakers & \\
\hline & Initial Position & middle Position & final Position \\
/I:/ & 7 & 8 & 6 \\
/i/ & 2 & 0 & 2 \\
/æ/ & 1 & 2 & 2 \\
\hline Total & 10 & 10 & 10 \\
\hline
\end{tabular}


The table-1 shows the pronunciation of the English front close target long vowel / r:/ at different conceivable positions in English words by Pashto speakers. Among the ten participants, only seven of them pronounced the target English front close target long vowel /r:/ correctly, at initial position of the word only. Out of the participants, only two of them pronounced the same long vowel sound as the short vowel /i/; while only one participant pronounced this sound like short vowel /æ/. Similarly, at word middle position, eight participants produced the English front close target long vowel / r:/ correctly as /r:/. None of them pronounced this long vowel as /i/, Likewise, only two participants pronounced this sound as the short vowel /æ/. Lastly, at final position of the word, six participants pronounced the English front close target long vowel / r:/ correctly as /r:/. Two of the participants pronounced this long vowel like the short vowel /i/, while two participants pronounced it as short vowel /æ/.

The table-2 shows the formant frequencies, i.e., F1 and F2 of the target English front close long vowel /i:/. The table shows that when Pashto speakers pronounced the front close long vowel /i:/, the formant frequencies for the pronunciation of this long vowel was different from the pronunciation of the long vowel by native speakers of English. The formant frequencies by non-native speakers at different positions of words were different from the mean formant frequencies of the long vowel sound /i:/, by native speakers.

Table 2: Mean formant frequencies by native and Pashto speakers for long vowel /I:/

\begin{tabular}{cccc}
\hline \multicolumn{2}{c}{ Mean formant frequencies of Long vowel /r:/ } & \multicolumn{2}{c}{ Average formant frequency of long vowels by } \\
by SSBE & Pashto Speakers \\
\hline F1/Hz & F2/Hz & F1/Hz & F2/Hz \\
296 & 2241 & 357 & 1914 \\
\hline
\end{tabular}

\subsection{The Pronunciation of English Close Back Long Vowel /u:/}

The following table-3 below shows the pronunciation of English close back target long vowel / u: /. This long vowel was pronounced at three different possible positions of English words. In the table, the leftmost column shows the different pronunciation (different realizations of a single long vowel) of the target long vowel / u:/. The rows in the table show the number of these realizations for this long vowel at different positions.

Table 3: Pronunciation of the close back target long vowel /u:/

\begin{tabular}{cccc}
\hline Pronunciation of target vowel /u:/ & \multicolumn{4}{c}{ The pronunciation of Pashto speakers } \\
\hline & Initial Position & Middle Position & Final Position \\
/u:/ & 8 & 6 & 6 \\
/o/ & 1 & 2 & 3 \\
/s:/ & 1 & 2 & 1 \\
\hline Total & 10 & 10 & 10 \\
\hline
\end{tabular}

The given table 3 above shows the pronunciation of the English close back target long vowel /u:/, at different conceivable positions in English words by Pashto speakers. Among the ten participants, only eight of them pronounced the target English close back target long vowel /u:/ correctly at word initial position only. One of the participants pronounced the same close back long vowel sound as the short vowel $/ \mho /$, while one participant pronounced it like the long vowel / $/ /$. Similarly, at word middle position, six participants pronounced the English close back target long vowel /u:/ correctly, while two participants pronounced it like short vowel / $\mho$ / and two other participants pronounced it as like long vowel / :/. 
Lastly, at the final position of the word, a total of six participants pronounced the English close back target long vowel /u:/ correctly as /u:/. Moreover, three of the participants pronounced this long vowel like the short vowel / $v /$, while only one participant produced this sound as long vowel / ๑:/.

The acoustic analysis of the selected English close back long vowel / u:/ pronounced by Pashto speakers at different positions of the word is given in the table-4. The value for this long vowel was compared with the standard value for this long vowel in Standard variety of British English. The column, on the leftmost side shows the mean frequencies of formants of the selected English close back long vowel / u:/ in the standard variety of British English. While the column, to the right, shows the mean frequency of the long vowel, at different possible position of word, by Pashto speaker of Yusafzai dialect.

Table 4: Mean formant frequencies by natives and Pashto speakers for long vowel/ $\mathrm{u}: /$

\begin{tabular}{cccc}
\hline \multicolumn{2}{c}{ Mean formant Frequencies of /u:/ by SSBE } & Average formant frequency of Pashto Speakers \\
\hline $\mathrm{F} 1 / \mathrm{Hz}$ & $\mathrm{F} 2 / \mathrm{Hz}$ & $\mathrm{F} 1 / \mathrm{Hz}$ & $\mathrm{F} 2 / \mathrm{Hz}$ \\
386 & 1587 & 386 & 1178 \\
\hline
\end{tabular}

\subsection{The Pronunciation of English Open Back Long Vowel / $\alpha$ :/}

The table- 5 below shows the articulation of English open back target long vowel / $\alpha$ :/. This long vowel was pronounced at three different possible positions of English words. In the table, the leftmost column shows the different pronunciation (different realizations of a single long vowel) of the target long vowel $/ \alpha$ :/. The rows in the table show the number of these realizations for this long vowel at different positions.

Table 5: Pronunciation of the English open back target long vowel / $\alpha$ :/

\begin{tabular}{lccc}
\hline Pronunciation of target vowel $/ \alpha: /$ & \multicolumn{4}{c}{ The pronunciation of Pashto speakers } \\
\hline & Initial Position & middle Position & final Position \\
$/ \alpha: /$ & 8 & 0 & 6 \\
$/ \Lambda /$ & 2 & 2 & 0 \\
$/ \mathrm{a} /$ & 0 & 8 & 4 \\
\hline Total & 10 & 10 & 10 \\
\hline
\end{tabular}

The given table-5 above shows the pronunciation of the English open back target long vowel $/ \alpha: /$ at different conceivable positions in English words by Pashto speakers. Among the ten participants, only eight of them pronounced the target English open back target long vowel / $\alpha$ : / correctly at word initial position only. Two of them pronounced the same long vowel sound as the short vowel $/ \Lambda /$, while none of the participant pronounced it like short vowel $/ \mathrm{a} /$. Similarly, at word middle position, none of the participants pronounced the English open back target long vowel / $\alpha$ : / correctly as / $\alpha$ :/. Two of the participants pronounced this long vowel as $/ \Lambda /$; likewise, eight participants pronounced this sound as the short vowel / o / at medial position of the word.

Lastly, at final position of the word, six participants pronounced the English open back target long vowel / $\alpha$ :/ correctly as / $\alpha$ : /. No one among the participants pronounced this long vowel like the short vowel / $\Lambda$ /, while four participants pronounced it as short vowel / a / at the final position of the word. 
The acoustic analysis of the selected English open back long vowel / $\alpha$ :/ pronounced by Pashto speakers at different positions of the word is given in the table 6 . The value for this long vowel is compared with the standard value for this long vowel in Standard variety of British English. The column, on the left side shows the mean frequencies of formants of the selected English open back long vowel / $\alpha$ : / in the standard variety of British English. While the column, to the right, shows the mean frequencies of the open back long vowel, at different possible position of word, by Pashto speaker of Yusafzai dialect.

Table 6: Mean formant frequencies by Pashto speakers and natives for long vowel / $\alpha$ :/

\begin{tabular}{cccc} 
Mean formant Frequencies of $/ \alpha$ :/ by & Average formant frequency of Pashto Speakers \\
SSBE & & \\
\hline $\mathrm{F} 1 / \mathrm{Hz}$ & $\mathrm{F} 2 / \mathrm{Hz}$ & 559 & $\mathrm{~F} 2 / \mathrm{Hz}$ \\
680 & 1193 & 1254 \\
\hline
\end{tabular}

\subsection{The Pronunciation of English Front Open-Mid Long Vowel /3:/}

The following table-7 shows the pronunciation of English front open-mid target long vowel / 3: /. This long vowel was pronounced at three different possible positions of English words. In the table, the leftmost column shows the different pronunciation (different realizations of a single long vowel) of the English front open-mid target long vowel / 3:/. The rows in the table show the number of these realizations for this long vowel at different positions.

Table 7: Pronunciation of the English front open-mid target long vowel / 3:/

\begin{tabular}{lccc}
\hline Pronunciation of target vowel /3:/ & \multicolumn{3}{c}{ The pronunciation of Pashto speakers } \\
\hline & Initial Position & middle Position & final Position \\
/3:/ & 4 & 6 & 3 \\
/r/ & 4 & 3 & 7 \\
/a/ & 2 & 1 & 0 \\
\hline Total & 10 & 10 & 10 \\
\hline
\end{tabular}

The table-7 shows the pronunciation of the English front open-mid target long vowel / 3: / at different conceivable positions in English words by Pashto speakers. Among the ten participants, only four of them pronounced the target English front open-mid target long vowel / 3: / correctly at word initial position only. Four of them pronounced the same long vowel sound as the short vowel / $\mathrm{r} /$, while two participants pronounced it like short vowel /a/. Similarly, at word middle position, six participants pronounced the English front open-mid target long vowel / 3:/ correctly as / 3:/. Three of them pronounced this long vowel as / $\mathrm{r} /$, Likewise, only one participant pronounced this sound as the short vowel / a /. Lastly, at final position of the word, only three participants pronounced the English front open-mid target long vowel / 3:/ correctly as / 3:/. Seven of the participants pronounced this long vowel like the short vowel / $\mathrm{r} /$, while no one pronounced it as short vowel /æ/.

The acoustic analysis of the selected English front open-mid long vowel /3:/ pronounced by Pashto speakers at different positions of the word is given in the table- 8 . The value for this long vowel is compared with the standard value for this long vowel in Standard variety of British English. The column, on the left side in the table shows the mean frequencies of formants of the selected English front open-mid long vowel / 3:/ in the standard variety of British English. While the column, to the right, shows the mean frequencies of the long vowel, at different possible position of word, by Pashto speaker of Yusafzai dialect. 
Table 8: Mean formant frequency by natives and Pashto speakers for long vowel / 3:/

\begin{tabular}{cccc}
\hline \multicolumn{2}{c}{ Mean formant Frequencies of /3:/ by SSBE } & Average formant frequency of Pashto Speakers \\
\hline F1/Hz & F2/Hz & F1/Hz & F2/Hz \\
519 & 1408 & 494 & 1357 \\
\hline
\end{tabular}

\subsection{The Pronunciation of English Close-Mid Back Long Vowel /s:/}

The following table-9 shows the pronunciation of English close-mid back target long vowel / ๑: /. This long vowel was pronounced at three different possible positions of English words. In the table, the left column shows the different pronunciation (different realizations of a single long vowel) of the close-mid back target long vowel / o:/. The rows in the table show number of these realizations for this long vowel at different positions.

Table 9: Pronunciation of English close-mid back target long vowel / o:/

\begin{tabular}{lccc}
\hline Pronunciation of target vowel / $/ / /$ & \multicolumn{3}{c}{ The pronunciation of Pashto speakers } \\
\hline & Initial Position & middle Position & final Position \\
/ :/ & 3 & 2 & 2 \\
/a:/ & 7 & 8 & 0 \\
/v/ & 0 & 0 & 8 \\
\hline Total & 10 & 10 & 10 \\
\hline
\end{tabular}

The table-9 shows the pronunciation of the English close-mid back target long vowel / o: / at different conceivable positions in English words by Pashto speakers. Among the ten participants, only three of them pronounced the target English close-mid back target long vowel / o: / correctly at word initial position only. Seven of them pronounced the same long vowel sound as the long vowel / a:/, while no one of participant pronounced it like short vowel / $\mathrm{J} /$. Similarly, at word middle position, only two participants pronounced the English close-mid back target long vowel / ๑:/ correctly as / ๑:/. Eight of them pronounced this long vowel as / a:/, Likewise, no one of the participants pronounced this sound as the short vowel / $v$ /. Lastly, at final position of the word, only two participants pronounced the English close-mid back target long vowel / o:/ correctly as / o:/. None of the participants pronounced this long vowel like the long vowel / a:/, while eight participants articulated this sound as short vowel / $v$ /.

The acoustic analysis of the selected English front close long vowel / o:/ pronounced by the Pashto speakers at different positions of the word is given in the table-10 below. The value for this long vowel is compared with the standard value in Standard variety of the British English. The column, on the left side shows the mean frequencies of the formants of the selected English front close long vowel / o:/ in the standard variety of the British English. While third column, to the right, shows the mean frequencies of the long vowel, at the different possible position of word, by Pashto speaker of Yusafzai dialect.

Table 10: Mean formant frequency by natives and Pashto speakers for long vowel / o:/

\begin{tabular}{cccc}
\hline \multicolumn{2}{c}{ Mean formant Frequencies of /o:/ by SSBE } & \multicolumn{2}{c}{ Average formant frequency of Pashto } \\
& & Speakers \\
\hline $\mathrm{F} 1 / \mathrm{Hz}$ & $\mathrm{F} 2 / \mathrm{Hz}$ & 528 & 1152 \\
480 & 857 & $5 \mathrm{~Hz}$ \\
\hline
\end{tabular}

The results of this research indicate that Pashto speaker of Yusafzai dialect learning English had difficulties in the accurate articulation of English long vowels. The problem arises due the 
phonological differences of both languages English and Pashto. Due to these phonological variances, the speakers of Pashto of Yusafzai dialect of English learning have either articulated the long vowels as near to English vowels or as vowels of Pashto. The speakers of Yusafzai dialect learning and speaking English articulated front close English long vowel /I:/ as open back vowel as /i/ and /æ/ and sometime they pronounced like Pashto vowels /ai/, /e/, /i/, and /I/. They have articulated the close back rounded English long vowel sound /u:/ as close long vowel of English like / $/$ / and back close rounded vowel sound as / / and sometime, they pronounced Pashto vowels like /ou/, and /ue/.

Pashto speaker of Yusafzai dialect learning English pronounced English open long vowel /a:/ as English open vowel sound $/ \Lambda /$ and /ə/, sometimes they pronounced it like Pashto vowels /i/, /ai/, and /æ/. They pronounced the English close back rounded long vowel sound /っ:/ as English open front long vowel /a:/ and close back rounded vowel / $/$, sometime the same vowel sound is pronounced like Pashto vowels /o/, /u/, / / and /ou/. Furthermore, the English mid-central long vowel /3:/ was pronounced as English central consonant sound / $\mathrm{x} /$ and English open front vowel sound /ə/, sometime they pronounced the same long vowel sound like Pashto sound /r/.

The results of this study revealed that Pashto speaker of Yusafzai dialect having background of English pronounced the targeted long vowels of English as a bit near to vowel sound of English or pronounced like native speakers. The root cause of this mispronunciation of the target long vowel by Pashto speaker of Yusafzai dialect learning English was due to the unfamiliarity with the target long vowel sounds. The problem of mispronunciation of word was due the orthographic combination of letters with sounds and the influence of mother tongue also played a pivotal role in the mispronunciation of the target long vowels.

\section{Conclusion}

The study investigated the English problematic long vowels. For this purpose, English words having the target long vowels were printed on a sheet of paper and the students were asked to pronounce these words. The data was collected through recording and later on was acoustically analyzed. The analysis of the data suggests that all the targeted English long vowels /I:/ /u:/, /a:/, /з:/, / ॰:/ were problematic for Pashto speakers of Yusafzai dialect. The participants from whom the data was collected did not pronounce the targeted vowels in a particular word correctly and precisely, as native speakers of English. They had mixed the pronunciation of target long vowels with different vowels of English or uttered them like Pashto vowels. They seemed to be unaware about English phonetic and phonological characteristics of long vowel sounds and so, as a result having no familiarity and idea about the phonology of English sounds. Every one of them utilized their very own way of pronunciation of the English long vowel sounds. The speakers of Pashto language had learned the way of articulation from their teachers having background of L1 (Pashto language) or such articulation had been affected by their L1 (Pashto language). Every language has a different types of speech sounds and different types of arrangement of these sounds.

When someone tries to convey his message in L2 (other language), he turns back to his/her L1 while uttering the speech sounds of L2. The root cause of the above problem is that L2 learners are not guided with appropriate way of articulation of the target language (English). Correct articulation of the target language is conceivable through interpretation, correlation and practice. On the other hand, the learners are not able to differentiate between sounds if they are not skilled in phonetics and phonology of L2 (target language). In terms of articulation of the 
word, particularly English subject and vocabulary should not be learnt or taught from orthographical perspective. There is no one to one correspondence between sounds and letter of a language. The incorrect articulation of target English long vowels determines that there are problematic long vowels which need attention to be improved. Moreover, appropriate attention and practice is required to students to overcome mispronunciation of the target language and they should be trained with the correct pronunciation.

\section{References}

Aronson, L. R., Rosenhouse, J., Rosenhouse, G., \& Podoshin, L. (1996). An acoustic analysis of modern Hebrew vowels and voiced consonants. Journal of Phonetics, 24 (2), 283293. https://doi.org/10.1006/jpho.1996.0015

Baidar, B., Rahman, G. \& Hamid, A. (2020). Acoustic analysis of English problematic short vowels for Pashto speakers. International Review of Social Sciences, 8(10), 124-138. https://irss.academyirmbr.com/papers/1602390410.pdf

Balas, A. (2018). English vowel perception by Polish advanced learners of English. Canadian Journal of Linguistics, 63(3), 309-338. https://doi.org/10.1017/cnj.2018.5

Benzeghiba, M., De Mori, R., Deroo, O., Dupont, S., Jouvet, D., Fissore, L., ... \& Tyagi, V. (2006, June). Impact of variabilities on speech recognition. In Conference Proceeding SPECOM (pp. 3-16), Saint-Petersburg, Russian Federation. https://www.eurasip.org/Proceedings/Ext/SPECOM2006/papers/001.pdf

Borzone-de-Manrique, A. M. (1979). Acoustic analysis of the Spanish diphthongs. Phonetica, 36(3), 194-206. https://doi.org/10.1159/000259958

Bohn, O. (2017). Cross-language and second language speech perception. In E. Fernandez and H. Cairns (Eds.). The Handbook of Psycholinguistics, (213-239). Wiley.

Borzone-de-Manrique, A. M. \& Massone, M. (1981). Acoustic analysis and perception of Spanish fricative consonants. Journal of Acoustic Society of America, 69(4), 11451153. https://doi.org/10.1121/1.385694

Bradlow, A. (1995). A comparative acoustic study of English and Spanish vowels. Journal of the Acoustical Society of America, 97 (3), 1916-1924. https://doi.org/10.1121/1.412064

Chladkova, K., Hamann, S., Williams, D., \& Hellmuth, S. (2017). F2 slope as a perceptual cue for the front-back contrast in standard Southern British English. Language and Speech, 60 (3), 377-398. https://doi.org/10.1177\%2F0023830916650991

Cebrian, J. (2019). Perceptual assimilation of British English vowels to Spanish monophthongs and diphthongs. The Journal of the Acoustical Society of America, 145(1), 52-58. https://doi.org/10.1121/1.5087645

Chen, Y. (2008). The acoustic realization of vowels of Shanghai Chinese. Journal of Phonetics, 36 (4), 629-648. https://doi.org/10.1016/j.wocn.2008.03.001

Chen, Y., Robb, M., Gilbert, H., \& Lerman, J. (2001). Vowel production by Mandarin speakers of English. Clinical Linguistics \& Phonetic, 15(6), 427-440. https://doi.org/10.1080/02699200110044804

Deterding, D. (1997). The formants of monophthong vowels in standard Southern British English pronunciation. Journal of International Phonetic Association, 27(1), 47-55. https://www.jstor.org/stable/44516138?seq=1

Deterding, D. (2003). An instrumental study of the monophthong vowels of Singapore English. English World Wide, 24 (1), 1-16. https://doi.org/10.1075/eww.24.1.02det 
Elvin, J., Williams, D., \& Escudero, P. (2016). Dynamic acoustic properties of monophthongs and diphthongs in Western Sydney Australian English. The Journal of the Acoustical Society of America, 140(1), 576-581. https://doi.org/10.1121/1.4952387

Gordon, R. J. (2005). Ethnologue: Languages of the world (15 ${ }^{\text {th }}$ Edition). SIL International.

Gordon, M., \& Ayla, A. (2006). Phonetic structures of Turkish Kabardian. Journal of the International Phonetic Association, $36 \quad$ (2), 159-186. https://www.jstor.org/stable/44526410?seq=1

Homma, Y. (1973). An acoustic study of Japanese vowels. their quality, pitch, amplitude, and duration. Study of Sounds $16, \quad 347-368$. https://www.scopus.com/record/display.uri?eid=2-s2.0$\underline{85050781701 \& \text { origin }=\text { inward\&txGid }=\mathrm{cc} 232769 \mathrm{c} 830 \mathrm{bcaa} 4588887 \mathrm{e} 63989 \mathrm{fc} 5}$

Jenkins, J. (2000). The phonology of English as an international language. Cambridge University.

Jia, Y., Wang, Y., Li, A., Song, D., \& Xu, L. (2017, October). Typology of convergences and divergences of English monophthongs by EFL learners from Guanhua regions. In National Conference on Man-Machine Speech Communication (pp. 36-46). Springer,Singapore. https://link.springer.com/chapter/10.1007/978-981-10-8111$\underline{8} 4$

Kent, R. D., \& Charles, R. (2002). The acoustic analysis of speech (2 ed.). Singular Publishing. Kewley-Porr, D., Akahane-Yamada, R., \& Aikaea, K. (1996). Intelligibility and acoustic correlates of Japanese accented English vowels. Fourth International Conference on Spoken Language: ICSLP-96, Vol. 1, 450-453. https://doi.org/10.1109/ICSLP.1996.607151

Lin, L.-C. (2014). Understanding pronunciation variations facing ESL students. International Journal of Humanities and Social Science, 5(1), 16-20. $\underline{\text { https://citeseerx.ist.psu.edu/viewdoc/download?doi=10.1.1.1040.5566\&rep=rep1\&t }}$ ype $=$ pdf

Mackenzie, D. N. (1959). A standard Pashto. Bulletin of the School of Oriental and African Studies, University of London, 22(1/3), 231-23. https://www.jstor.org/stable/609426?seq=1

Maxwell, O. \& Janet, F. (2009). Acoustic and durational properties of Indian English vowels. World Englishes, 28(1), 52-69. https://doi.org/10.1111/j.1467-971X.2008.01569.x

Mixdorff, H., Cole, J., \& Shattuck-Hufnagel, S. (2012). Prosodic similarity: evidence from an imitation study. $6^{\text {th }}$ International Conference on Speech Prosody (pp. 571-574). Tongji University. https://www.iscaspeech.org/archive/sp2012/papers/sp12 571.pdf

Moore, B. C. (2003). An introduction to the psychology of hearing (5 ed.). Emerald Group.

Moulton, W. G. (1962). Toward a classification of pronounciation errors. The Modern Language Journal, 46(3), 101-109. https://www.jstor.org/stable/320931?seq=1

Peterson, G. E., \& Barney, H. L. (1952). Control methods used in a study of the vowels. Journal of the Acoustical Society of America, 24(2), 175-184. https://doi.org/10.1121/1.1917300

Prica, B., \& Ilić, S. (2010). Recognition of vowels in continuous speech by using formants. FACTA Universitatis-Series: Electronics and Energetics, 23(3), 379-393. https://doi.org/10.2298/FUEE1003379P

Rahman, G. (2009). A comparative study of Pashto and English phonology for English language teaching and learning. Master's Thesis, University of Management \& Technology, Lahore. 
Rahman, T. (2002). Language ideology and power: language learning among the Muslims of Pakistan and North India. Oxford University. https://doi.org/10.1353/lan.2004.0149

Rehman, G., Khan, A. Q., \& Bukhari, N. H. (2012). English problematic consonants for Pashto speakers. Academic Research International, 2(1), 793-797. http://www.savap.org.pk/journals/ARInt./Vol.2\%281\%29/2012\%282.1-68\%29.pdf

Roach, P. (2000). English phonetics and phonology. Cambridge University.

Roettger, T. B., Winter, B., \& Baayen, H. (2019). Emergent data analysis in phonetic sciences: towards pluralism and reproducibility. Journal of Phonetics, 73, 1-7. https://doi.org/10.1016/j.wocn.2018.12.001

Sethi, J., \& Dhamija, P. V. (1999). A course in phonetics and spoken English. Prentice-Hall of India.

Schaetzel, K. \& Low, E. L. (2009, July). Teaching pronunciation to adult English language learners. CAELA Network Brief. https://files.eric.ed.gov/fulltext/ED540593.pdf

Schwartz, G., Aperlinski, G., Jekiel, M., \& Malarski, K. (2016). Spectral dynamics in L1 and L2 vowel perception. Research in Language, 14(1), 61-77. https://doi.org/10.1515/rela-2016-0004

Schwartz, G., \& Kaźmierski, K. (2019). Vowel dynamics in the acquisition of L2 English: an acoustic study of L1 Polish learners. Language Acquisition, 27(3), 227-254. https://doi.org/10.1080/10489223.2019.1707204

Slabakova, $R$. (2016). Second language acquisition. Oxford University.

Swan, M., \& Smith, B. (2001). Learner English: a teacher's guide to interference and other problems. Cambridge University.

Ouni, S., \& Yves, L. (2003). A study of the French vowels through the main constriction of the vocal tract using an acoustic-to-articulatory inversion method. In the $15^{\text {th }}$ International Congress of Phonetic Sciences, 2901-2904. Barcelona. https://hal.inria.fr/inria-00107719/

Yuasa, I. (2001). Politeness, emotion and gender: a socio-phonetic study of voice pitch modulation. Doctoral dissertation, University of California, Berkeley. 


\section{Annexure:}

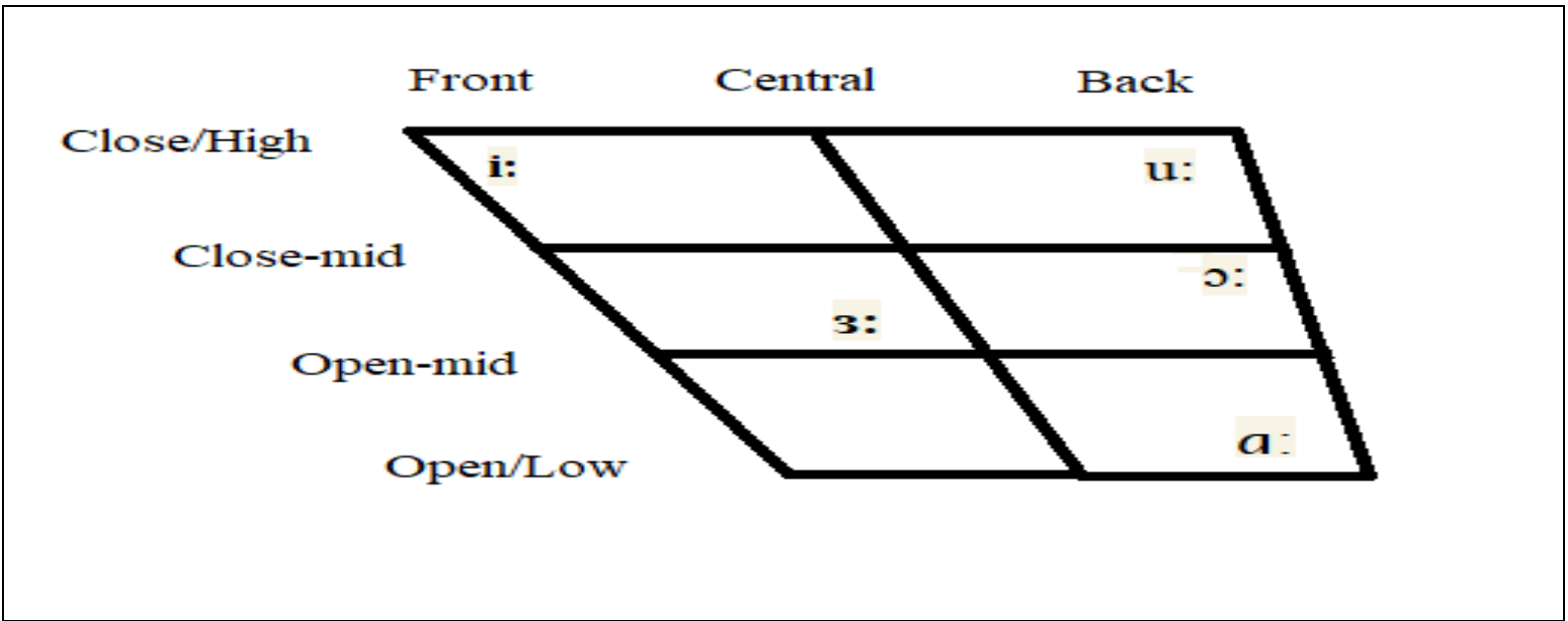

Figure 1: Long Vowels (Roach, 2000)

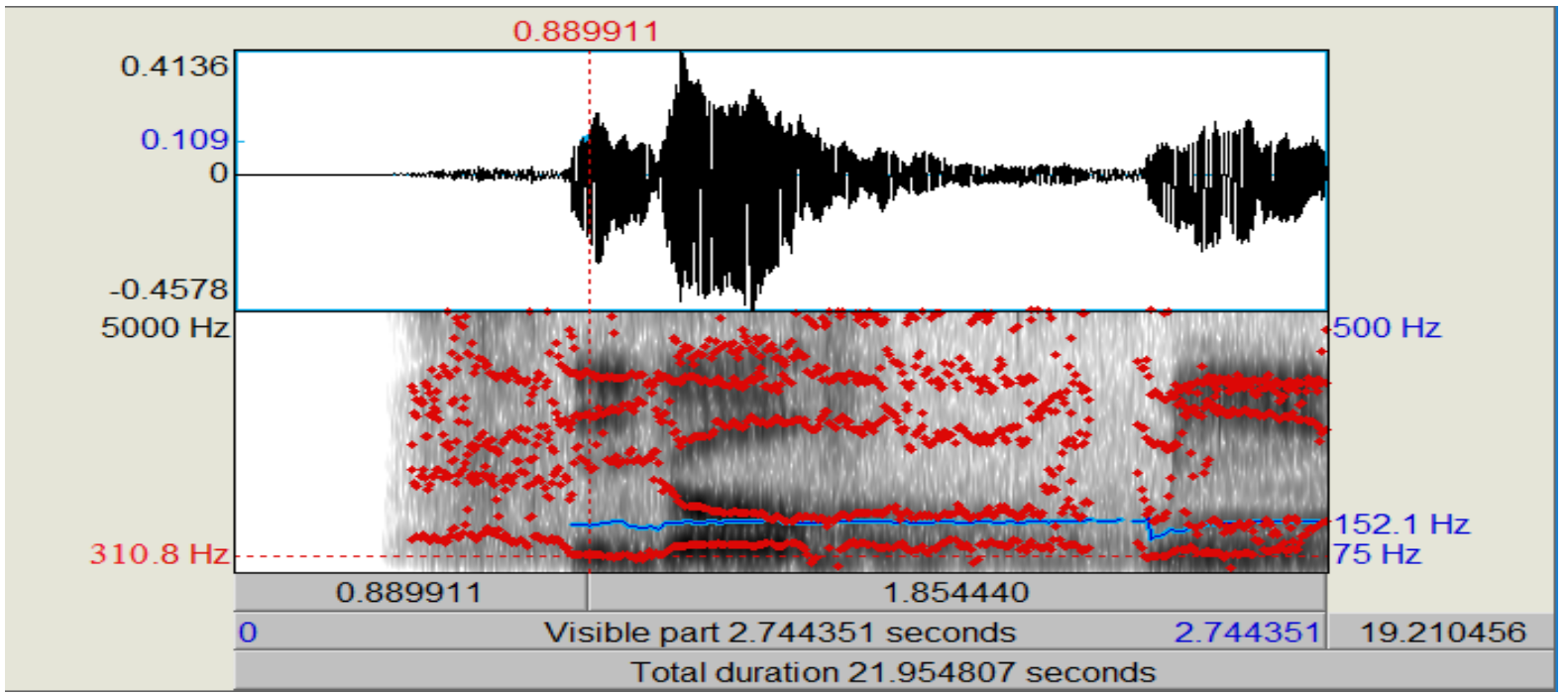

Figure 2: Spectrogram of English front close long vowel sound / i: /

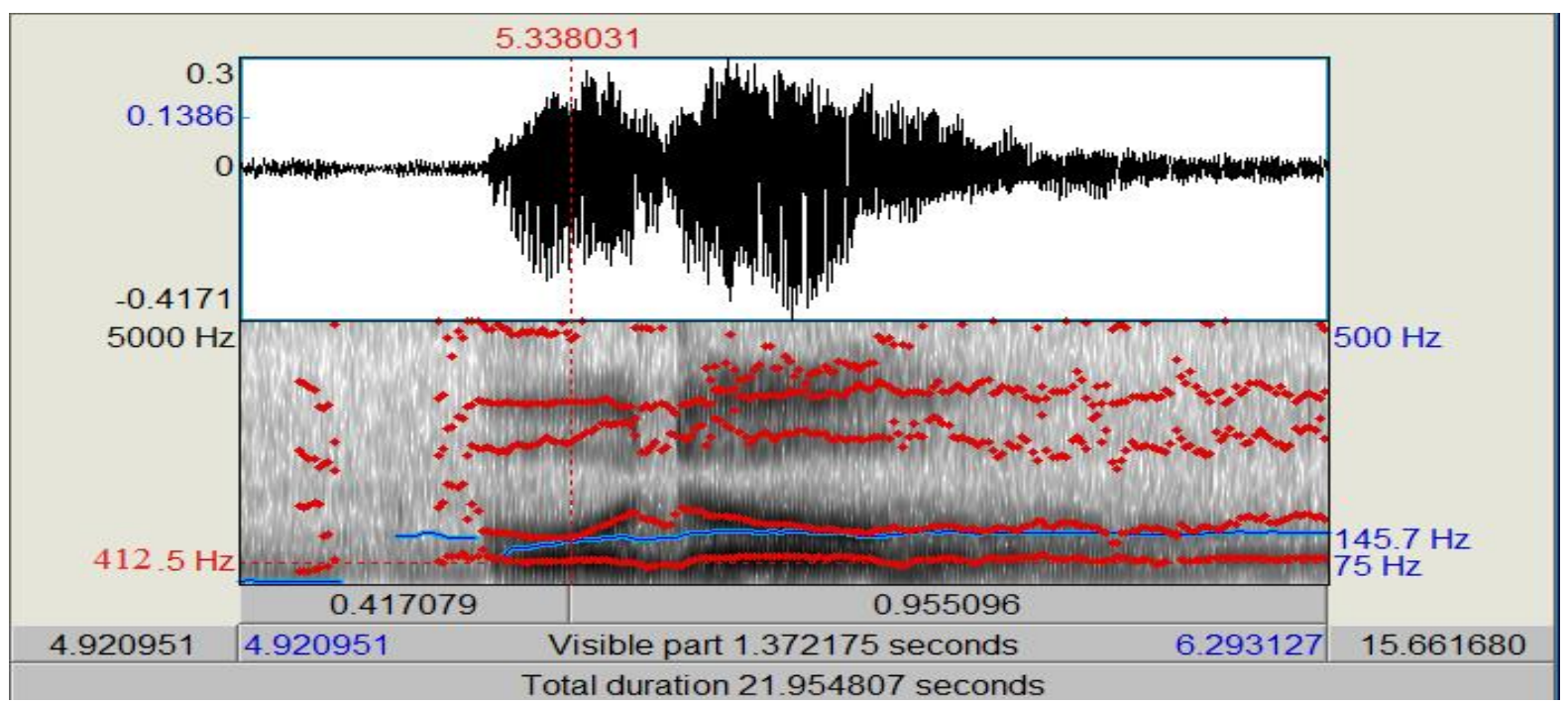

Figure 3: Spectrogram of target close back long vowel /u:/ 


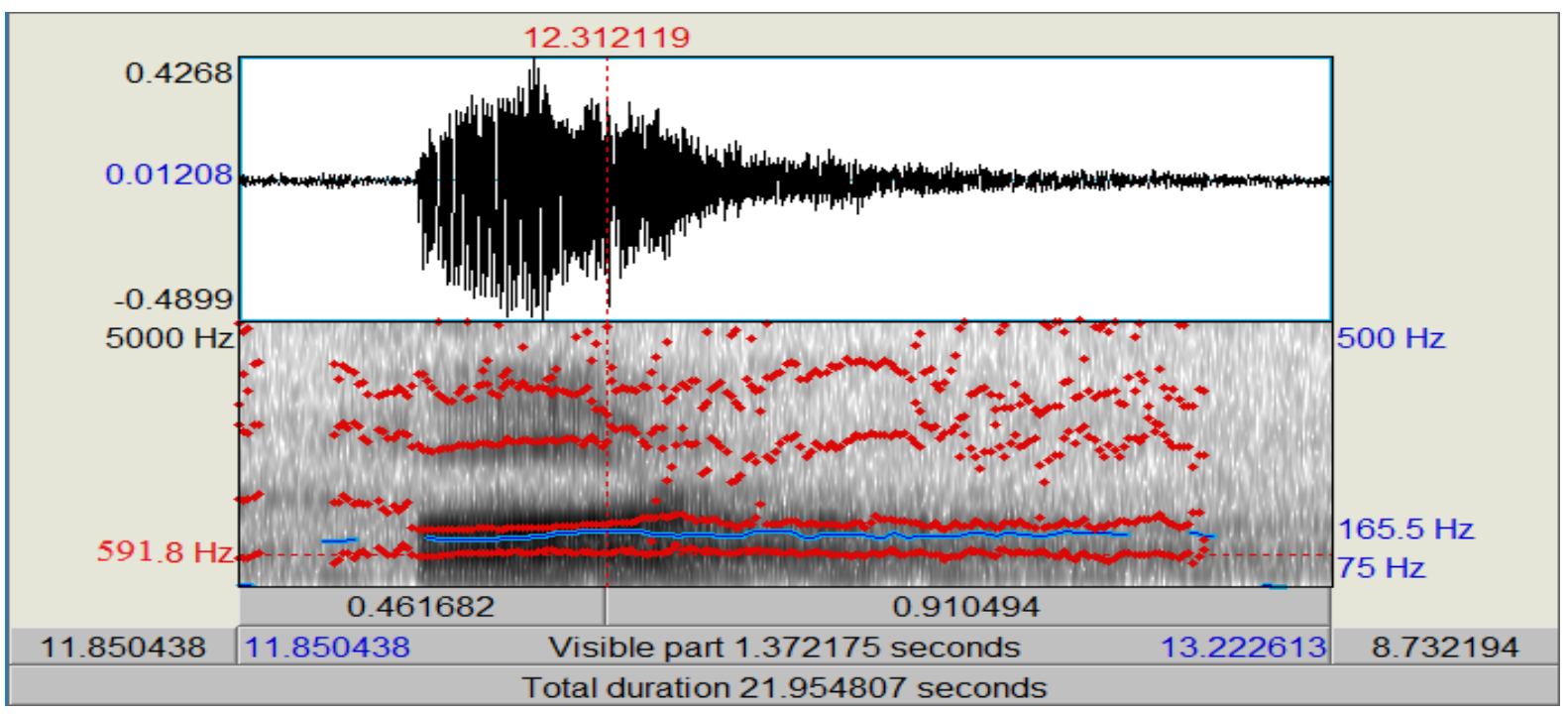

Figure 4: The pronunciation of open back of long vowel $/ \alpha$ :/

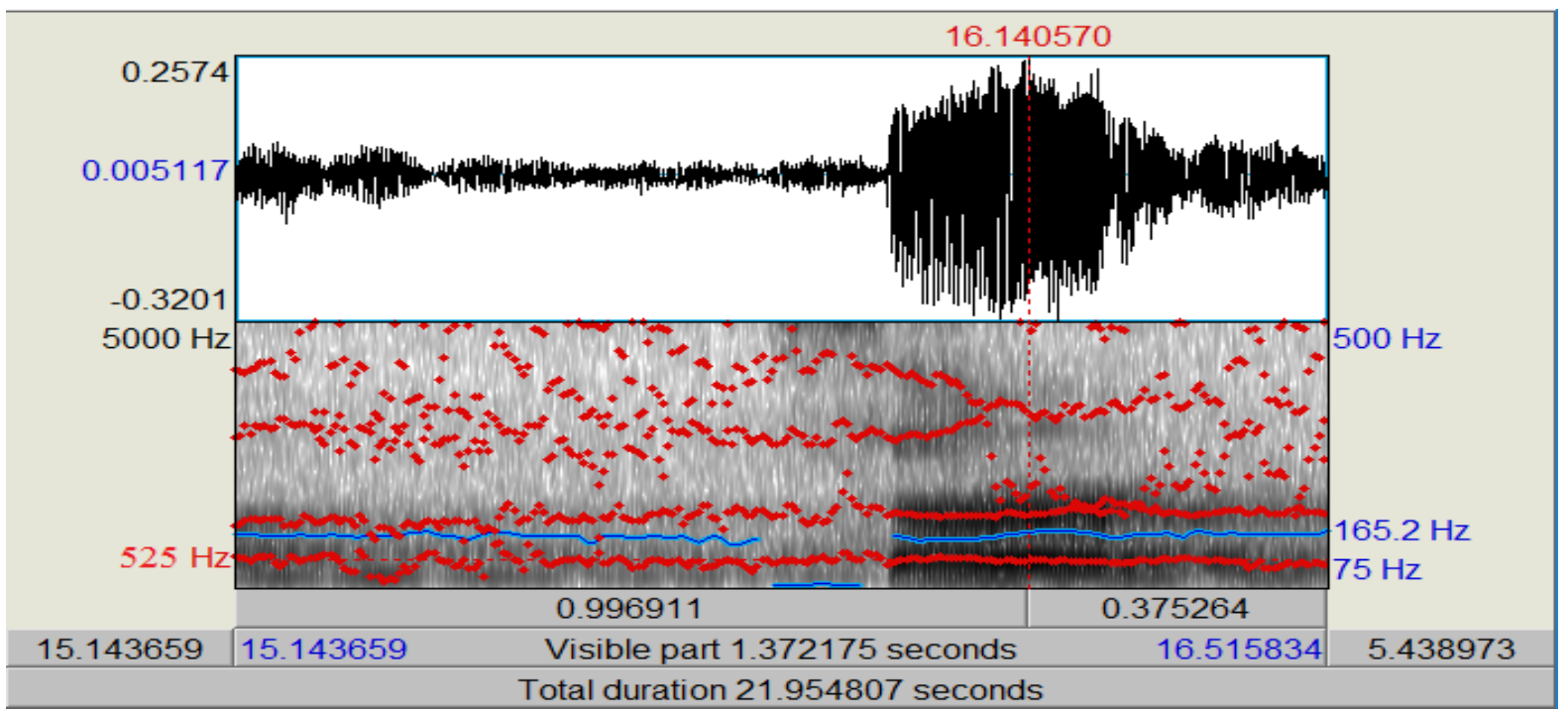

Figure 5: The pronunciation of front open-mid long vowel /3:/

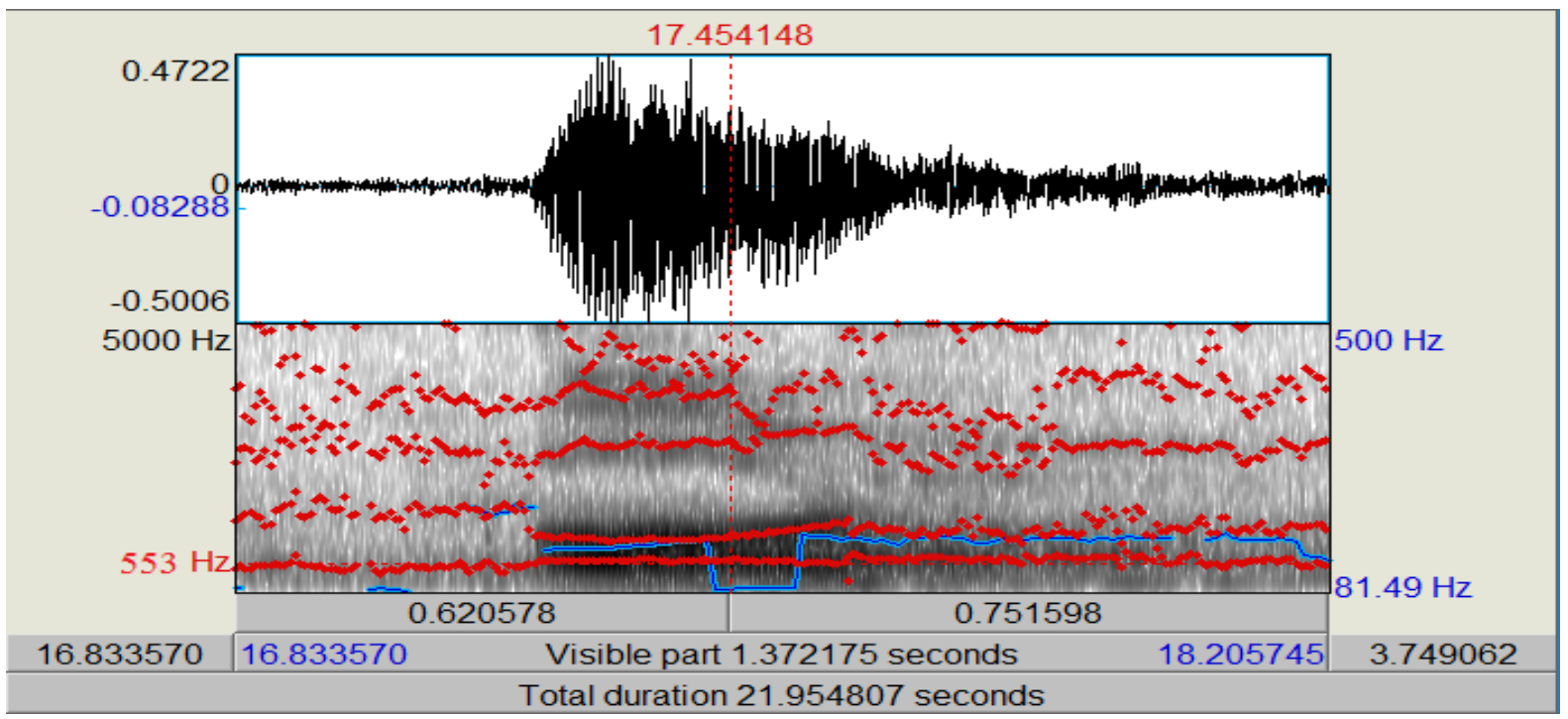

Figure 6: The pronunciation of close-mid back long vowel /s:/ 